\title{
Modeling of the rheological properties of multinanolayer films in the presence of compatibilized interphase
}

\author{
Q. Beuguel, ${ }^{\text {a) }}$ A. Guinault, F. Chinesta, C. Sollogoub, and G. Miquelard-Garnier ${ }^{\text {a) }}$ \\ Laboratoire PIMM, Arts et Metiers Institute of Technology, CNRS, CNAM, Hesam University, \\ 151 Boulevard de l'Hôpital, 75013 Paris, France
}

\begin{abstract}
Rheological behavior of nanolayered films of polyethylene/polyamide 6 (PE/PA6) compatibilized in situ during the coextrusion process has been studied at a temperature between the melting temperatures of PE and PA6. Thanks to the high number of interfaces, a drastic increase in dynamic moduli has been measured when increasing the interphase volume fraction in the films, and a solid-like behavior for the interphase was identified. Different models are compared to capture the complex viscosity of nanolayered films as a function of angular frequency. A model considering interphase and bulk viscosities and a single fitting parameter, namely, the thickness over which the viscosity decreases linearly from the interphase to the bulk one, captures the complex viscosity of all samples. This thickness is comparable to the PE layer thickness up to values about $1 \mu \mathrm{m}$ before a significant bulk region has to be added to capture the behavior for thicker layers. This suggests that the melt rheology is impacted by the presence of a nanometric interphase over very large (micronic) length scales.
\end{abstract}

\section{INTRODUCTION}

A well-known strategy in the industry to improve the final properties of materials resulting from the combination of two immiscible polymers is the addition of a third component (named compatibilizer in the case of polymer blends or tie layer in the case of coextruded polymers) [1,2]. This third component is usually a copolymer, formed ex situ or in situ, which presents chemical affinities with both phases and will thus segregate preferentially at the interfaces.

By doing so, it will lead to the formation of an interphase, i.e., a region with singular properties, having a thickness comparable to the typical copolymer size ( $\gtrsim 10 \mathrm{~nm}$ ) [3]. This interphase will lead to a decrease in interfacial tension between the immiscible polymers [4] and will promote interfacial adhesion through entanglements with bulk chains, preventing, for example, delamination [5]. The impact of the interphase on interfacial adhesion has been studied for the last 30 years and quantitative macromolecular mechanisms have been proposed. They show this adhesion is controlled by the compatibilizer density at the interface and its interpenetration with bulk chains [6,7]. However, the intrinsic rheological or mechanical properties of these interphases are difficult to measure or extract from the materials' ones, due to the low volume fraction of the interphase.

The coextrusion process of multinanolayered films can increase the number of layers in coextruded systems up to a few thousands while decreasing their thickness down to few nanometers [8,9] simultaneously, with the use of a large number $N(\sim 10)$ of layer multiplying elements (LMEs). In

\footnotetext{
a) Authors to whom correspondence should be addressed; electronic mail: quentin.beuguel@wanadoo.fr and guillaume.miquelardgarnier@lecnam.net
}

multinanolayered films, the layer thickness becomes then comparable to the interphase one. Moreover, in such systems, the multilayer structure is maintained, contrary to compatibilized polymer blends where the blend morphology (i.e., size and shape of the dispersed phase) is also impacted by the presence of a compatibilizer. The high number of interfaces then amplifies drastically the role of compatibilized interphase on adhesion and failure mechanisms [10] by increasing the volume fraction of the interphase. This allows to study specifically the role of interphases on the final properties, using conventional tools of polymer analysis and considering a well-controlled interface geometry.

It has long been known that rheology is an efficient tool to probe the role of interfaces in polymer blends [11-13]. In these studies, the appearance of an additional relaxation time has been related to the presence of an interphase in compatibilized thermoplastic blends, due to polymer chain relaxation delay. Rheology has also been shown to be an efficient probe of interfaces in multilayered coextruded films. In extensional rheometry, a strain hardening behavior, related to reactions occurring at the interface, has been evidenced by several authors [14-16]. In shear rheometry, Zhao and Macosko [17] observe that the addition of a reactive compatibilizer in multilayer films suppresses the interfacial slip under shear between immiscible polymer layers.

Building on these approaches, we recently took advantage of multinanolayer compatibilized polyethylene (PE)/polyamide 6 (PA6) films with hundreds to thousands of interfaces (i.e., high interfacial area) to rheologically study the interphases by analyzing the films' linear viscoelastic properties. A solid-like behavior has been reported for multinanolayer compatibilized films [18], and the rheological properties of the interphase were also quantitatively measured. We demonstrated a synergetic effect between the compatibilized 
interphase and the bulk. The apparent complex viscosity of multilayer films with various number of layers has been qualitatively captured using a simple weighted mixing law of bulk and interphase viscosities over a large angular frequency range [18].

The aim of this study is to go further on the analysis of compatibilized interphases and their effect on the material rheological properties. Building on the same experimental protocol, we here develop rheological models with various viscosity profiles to fit more quantitatively the experimental behavior of such multinanolayer systems. The models are then compared and discussed, as they reveal an influence of the interphase that extends over long (micronic) distances.

\section{EXPERIMENTAL PART}

\section{A. Materials}

A linear low-density polyethylene (PE, Dowlex ${ }^{\circledR} 2645$, Dow Chemical Company, Midland, USA) and a polyamide 6 (PA6, Ultramid® B40, kindly provided by BASF, Ludwigshafen, Germany) were used for multilayer film preparation. A linear low-density polyethylene-graft-maleic anhydride (PEgMA, Amplify® TY1353, Dow Chemical Company, Midland, USA) with a low graft level of $<0.25$ wt. \% (as given in the technical datasheet) was used as a compatibilizer. The analysis of the FTIR spectrum obtained in transmission for a pure PEgMA film (using a FTIR Frontier by Perkin-Elmer with a $4 \mathrm{~cm}^{-1}$ resolution and 16 scans) following the protocol described in Ref. [19] gives a grafting level in the $0.14-0.18 \mathrm{wt}$. \% range, consistent with the technical datasheet. Even at these low concentrations, compatibilization is effective: Garmabi et al. showed, for example, that the interfacial tension in PEgMA/PA6 blends decreases sharply from $12 \mathrm{mN} / \mathrm{m}$ to less than $2 \mathrm{mN} / \mathrm{m}$ with a saturation occurring at about 0.2 wt. \% MA [20].

The main characteristics of these three polymers were reported in a previous study [18]. Notably, we have $M_{w}=122 \mathrm{~kg} / \mathrm{mol}$ and $M_{n}=24 \mathrm{~kg} / \mathrm{mol}$ for PE and $M_{w}=85 \mathrm{~kg} / \mathrm{mol}$ and $M_{n}=21 \mathrm{~kg} / \mathrm{mol}$ for PEgMA. Due to its hydroscopic nature, PA6 was dried $48 \mathrm{~h}$ at $80^{\circ} \mathrm{C}$ under a dry air flow prior to extrusion to avoid thermal degradation during processing. The rheological properties of PE, PEgMA, and PA6 at the extrusion temperature $\left(240^{\circ} \mathrm{C}\right)$ are presented in Fig. S1 [37].

\section{B. Film preparation and morphological characterization}

Multilayer films were processed using the coextrusion line schematized in Fig. 1(a). The initial five-layer (PE/PEgMA/ PA6/PEgMA/PE) melt flow passed successively through $N=0,5,8$, or 9 LME, which sliced it vertically and recombined it horizontally, allowing the multiplication of the layer number from 5 to 2049. The films were cooled down on chill rolls thermoregulated at $T=80{ }^{\circ} \mathrm{C}$ and stretched to a final film thickness of $z \sim 25$ or $100 \mu \mathrm{m} \pm 10 \%$. The flow rates were fixed to $25 / 50 / 25,33 / 34 / 33,25 / 25 / 50,16.5 / 16.5 / 67$, and $0 / 33 / 67 \mathrm{wt} . \%$, leading to a total mass flow ranging from 1.2 to $1.7 \mathrm{~kg} / \mathrm{h}$, depending on the concentrations. For such mass flows, the shear rate was close to $7 \mathrm{~s}^{-1}$ in the LME and
$4 \mathrm{~s}^{-1}$ in the sheeting die while the total residence time is estimated between 80 and $120 \mathrm{~s}$ [21], sufficient to ensure PEgMA grafting on $\mathrm{NH}_{2}$ end groups of PA6 [22]. PE and PEgMA are miscible polymers and were considered merged in one layer (see also Sec. III) having a theoretical thickness, estimated from processing parameters, ranging from $x_{\text {calc } \mathrm{PE} / \mathrm{PEgMA}}=38180$ to $17 \mathrm{~nm}$ for different systems. ${ }^{1}$ For all the films, the ratio between the available number of PEgMA chains and the maximum surface density of interfacial PA6 chains is always much larger than 1. In the following, we then assume that the interphase is quasisaturated and has a constant thickness, identical for all the films [18].

Submicrometer scale observations were performed using a multimode atomic force microscope (AFM) controlled by a Veeco (Plainview, USA) Nanoscope V controller under ambient atmosphere [18]. The measured average thickness of PE/PEgMA layers $x_{\text {meas }}$ PE/PEgMA and its standard deviation $s t d_{\text {PE/PEgMA }}$ values were obtained by averaging the measurement of at least $10 \%$ of the total number of layers using several images $(\sim 5)$ taken at different locations in the films [23].

The multilayered morphologies of 25/50/25 [Fig. 1(b)], 33/34/33 [Fig. 1(c)], 16.5/16.5/67 [Fig. 1(d)], and 0/33/67 [Fig. 1(e)] wt. \% PE/PEgMA/PA6 coextruded multilayer films using $N=8 \mathrm{LME}$ and having a thickness of $z \sim 100 \mu \mathrm{m}$ are presented. The measured average thickness of PE/ PEgMA layers $x_{\text {meas }}$ PE/PEgMA is close to the theoretical one (Table I). Its standard deviation $s t d_{\mathrm{PE} / \mathrm{PEgMA}}$ highlights a relatively high layer thickness dispersion, probably due to viscosity ratios between polymers higher than $1[18,21]$. Complementary measurements of PE/PEgMA layer thicknesses for other systems are available in Table S2 [37].

\section{Rheological characterization}

Controlled stress rheometer MCR 502 (Anton Paar, Graz, Austria), equipped with a plate/plate geometry, was used in order to perform the linear viscoelastic tests, at $T=180^{\circ} \mathrm{C}$, on stacked multilayer films: 16 (for $z \sim 100 \mu \mathrm{m}$ ) or 32 (for $z \sim 25 \mu \mathrm{m})$ multilayer films were stacked and slightly compressed $\left(\sim 1.10^{3} \mathrm{~Pa}\right)$ under vacuum at $140{ }^{\circ} \mathrm{C}$ for $30 \mathrm{~min}$ to enable PE and PEgMA chains relaxation. It was verified that there is no influence of the stacked films number on the rheological properties (see Fig. S3 [37]) [18]. The rheological properties of the multilayer films were compared to those of PE/PEgMA bulk (50/50, 33/67, or $0 / 100$ wt. \%, depending on film concentrations), prepared in the same conditions, from mixed and compressed pellets. Strain sweep tests, with strain amplitude $\gamma$ ranging from 0.0001 to 1 , at $\omega=1$ rad.s ${ }^{-1}$, and frequency sweep tests, with angular frequency $\omega$ ranging from $10^{2}$ to $10^{-2}$ rad.s ${ }^{-1}$, in the linear domain (at strain $\gamma_{O}=0.01$ ), were performed. The thermal stability of the multilayers' rheological response over the experimental time has been carefully verified and thoroughly discussed in our previous work [18].

\footnotetext{
${ }^{1} x_{\text {calc }}$ was noted as $x_{t h}$ in Ref. [17].
} 

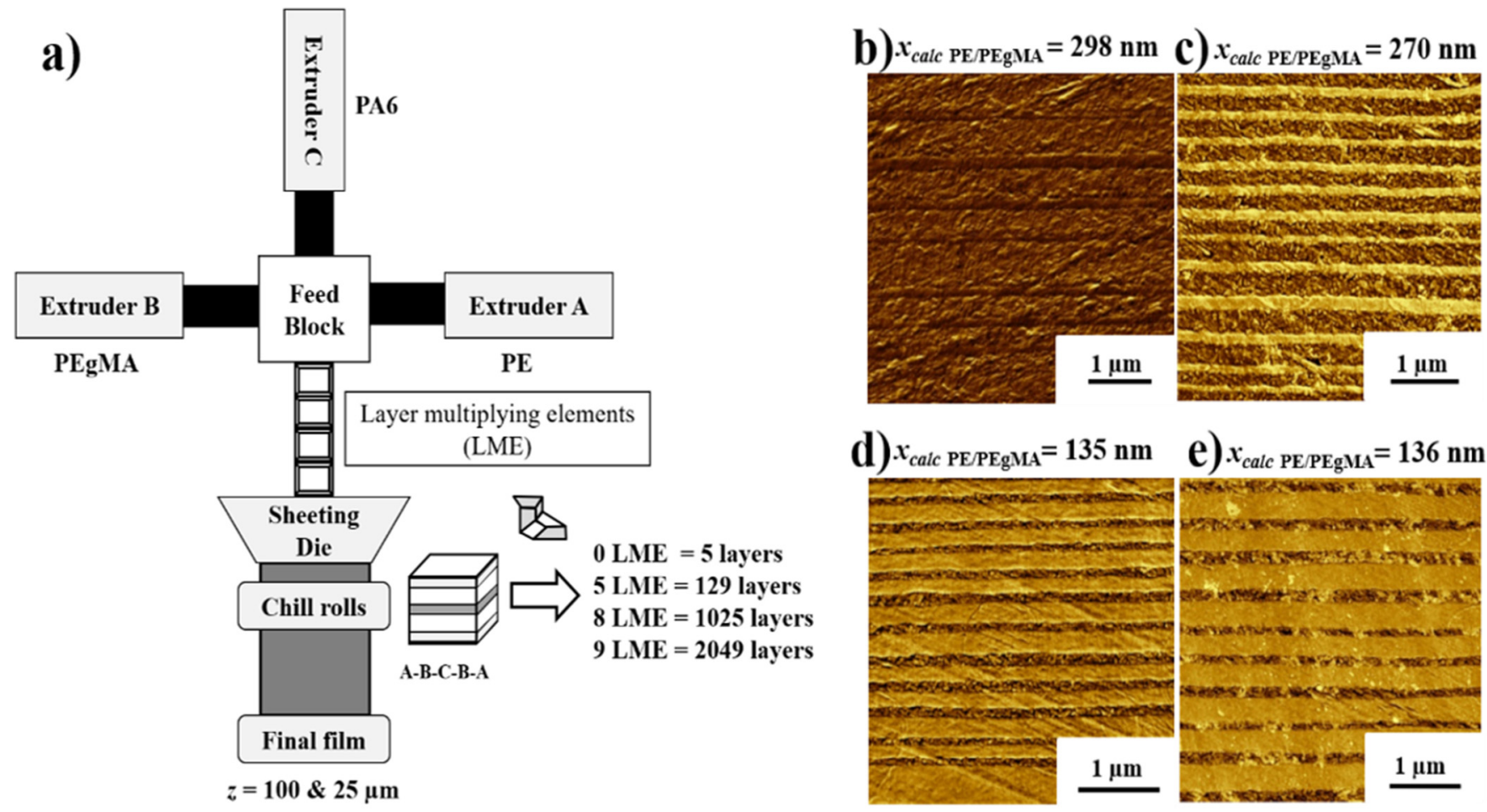

FIG. 1. Schematic of the coextrusion process (a) and AFM images (b)-(e) of 8 LME $100 \mu \mathrm{m}$ for 25/50/25 (b), 33/34/33 (c), 16.5/16.5/67 (d), and 0/33/ 67 wt. \% (e) PE/PEgMA/PA6 multilayer films.

\section{RESULTS AND DISCUSSION}

Within the extrusion timeframe, the maleic anhydride groups of PEgMA react with the amine end groups of PA6. The low amount of MA leads to typically one MA group per PEgMA. As a first approximation, it can be assumed that PE-PA6 Y-shaped copolymers are formed at the interfaces between PE and PA6 layers [22]. It is worth noting that quantifying the grafting density is not an easy task. It has been done on coextruded polyolefine/polyamide bilayer films using XPS [22] or more recently FTIR, involving a relatively complex step of selective dissolution of the PA layer [24]. To the best of our knowledge, this has never been achieved on multilayer films: it is not clear how a multilayer structure will react to the selective dissolution step, especially with $\mathrm{PE}$ being the outer layers.

Still, since for all systems studied, PEgMA is in large excess compared to available PA6 at the interface (see [18] for a more detailed discussion) and the processing time is sufficient for compatibilization to occur [22], it can be reasonably assumed that the interphase thickness is identical for

TABLE I. Dimensions of PE/PEgMA layers in PE/PEgMA/PA6 multilayer films presented in Fig. 1.

\begin{tabular}{lccc}
\hline \hline $\begin{array}{l}\text { Fraction } \\
(\text { wt. } \%)\end{array}$ & $\begin{array}{c}x_{\text {calc }} \text { PE/PEgMA } \\
(\mathrm{nm})\end{array}$ & $\begin{array}{c}x_{\text {meas }} \text { PE/PEgMA } \\
(\mathrm{nm})\end{array}$ & $\begin{array}{c}s t d_{\mathrm{PE} / \mathrm{PEgMA}} \\
(\mathrm{nm})\end{array}$ \\
\hline $25 / 50 / 25$ & 298 & 400 & 120 \\
$33 / 34 / 33$ & 270 & 200 & 70 \\
$16.5 / 16.5 / 67$ & 135 & 160 & 60 \\
$0 / 33 / 67$ & 136 & 130 & 50 \\
\hline
\end{tabular}

all samples, i.e., the end-to-end distance of the PE-PA6 copolymer.

At $T=180^{\circ} \mathrm{C}$, PA6 is in the solid state while PE and PEgMA are in the molten state. Since both PE and PEgMA are miscible and undiscernible in the AFM images (Fig. 1), they are considered in a first approach as a homogeneous blend in the following.

At this temperature, the compatibilized PE/PEgMA/PA6 multilayer system can then be described as a multilayer of the PE/PEgMA melt which is at the interfaces interpenetrated with a Y-shaped PE brush anchored on a PA6 solid surface. The typical thickness of this anchored PE is $x_{\mathrm{i}} \sim 15 \mathrm{~nm}$ (see calculation details in Ref. [18]) (see Fig. 2).

The stress transfer over the total sample thickness is assumed, based on a no slip condition reported by Zhao and Macosko [17] for similar multilayer compatibilized systems. Under shear, solid PA6 can be viewed as a wall that only reduces the volume sheared (see Sec. III B). In the middle of the PE/PEgMA layer, PE/PEgMA bulk properties are considered over a thickness $2 x_{\mathrm{b}}$. A transition thickness $x_{\mathrm{x}}$ from the interphase to bulk properties in the PE/PEgMA layer will be used as an adjusting parameter in the following models. We assume the interphases are identical so the system is symmetric at $x=0$ (Fig. 2). Note that since we only consider the PE phase, $x_{\mathrm{i}}$ defines only the PE-side of the interphase and not its total thickness (which would be the copolymer end-to-end distance, about $30 \mathrm{~nm}$ ).

\section{A. Rheological properties}

Figure 3(a) presents the variation of the elastic modulus $G^{\prime}$ as a function of strain $\gamma$ for PE/PEgMA bulk 


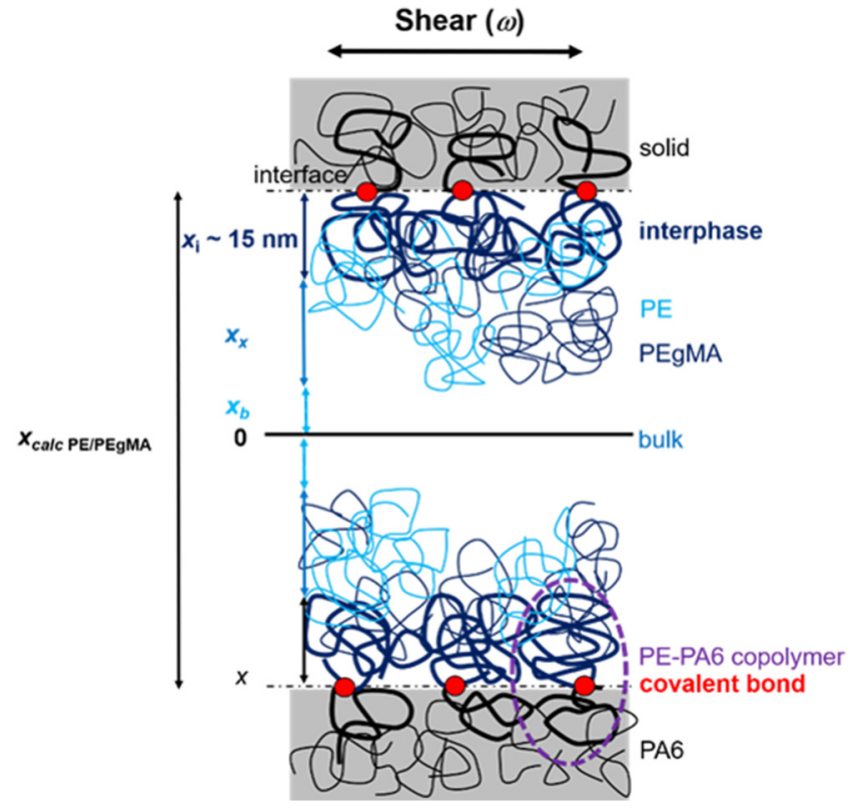

FIG. 2. Schematic of an inner layer of the compatibilized PE/PEgMA/PA6 film.

(33/67 wt. \%) and 25/50/25 wt. \% PE/PEgMA/PA6 multilayer films ( $x_{\text {calc }}$ PE/PEgMA ranging from 38180 to $17 \mathrm{~nm}$ ). The $0 / 33 / 67$ wt. $\%$ system characterized by $x_{\text {calc }}$ PEgMA $=17 \mathrm{~nm}$, which we consider as a pure interphase system [18], is also shown. The critical deformation $\gamma_{c}$, determined at the 5\% reduction of $G^{\prime}$ [25] [Fig. 3(a)], characterizing the limit between the linear and nonlinear domain, is plotted in Fig. 3(b) as a function of interphase volume fraction $\phi_{\mathrm{i}}$ for all PE/PEgMA/PA6 film concentrations. The interphase volume fraction is defined as in the following equation:

$$
\phi_{\mathrm{i}}=\frac{x_{\mathrm{i}}}{x_{\mathrm{i}}+x_{\mathrm{x}}+x_{\mathrm{b}}} .
$$

Due to the merging of the external layer after each LME and the symmetric structure of the PE/PEgMA layer (Fig. 2), $x_{\text {calc }}$ PE/PEgMA layer is defined as follows:

$$
\begin{array}{ll}
x_{\text {calc PE/PEgMA }}=x_{\mathrm{b}}+x_{\mathrm{x}}+x_{\mathrm{i}} & \text { for } \quad
\end{array}
$$

where $x_{\mathrm{b}}, x_{\mathrm{x}}$, and $x_{\mathrm{i}}=15 \mathrm{~nm}$ are the bulk, transition (from bulk to interphase viscosity), and interphase thicknesses, respectively.

The elastic plateau $G_{p}^{\prime}$ at low strain of all multilayer films is higher than that of PE/PEgMA bulk (33/67 wt. \%) [Fig. 3(a)]. $G_{p}^{\prime}$ increases from 3600 to $250000 \mathrm{~Pa}$ coupled with a decrease in the critical strain $\gamma_{c}$ from $40 \%$ to $0.9 \%$ for interphase volume fraction $\phi_{\mathrm{i}}$ ranging from $\sim 4 \times 10^{-4}$ to $\sim 1$. This decrease in $\gamma_{c}$ can be roughly described by a power law of the interphase volume fraction with an exponent close to -0.5 [Fig. 3(b)]. Nonlinearity appears at lower strain in the case of thin films (i.e., high interphase volume fraction).

The raw data of elastic $G^{\prime}$ and loss $G^{\prime \prime}$ moduli as a function of angular frequency $\omega$, in the linear domain $\gamma<\gamma_{c}$, are available in Fig. S4 [37]. The evolution of the relative elastic $G_{r}^{\prime}$ [Fig. 3(c)] and loss ${G^{\prime \prime}}_{r}$ [Fig. 3(d)] moduli, defined as the ratio between the modulus of the multilayer film over the PE/ PEgMA bulk one as a function of the interphase volume fraction $\phi_{\mathrm{i}}$ (at $\omega=0.01 \mathrm{rad} . \mathrm{s}^{-1}$ ), is presented. As mentioned for the critical strain $\gamma_{c}$ [Fig. 3(b)], the increases of both relative elastic $G_{r}^{\prime}$ [Fig. 3(c)] and loss ${G^{\prime \prime}}_{r}$ [Fig. 3(d)] are controlled by the interphase volume fraction for all PE/PEgMA/PA6 film concentrations. More precisely, at $\omega=0.01 \mathrm{rad} . \mathrm{s}^{-1}$, the increase of $G_{r}^{\prime}$ is $\sim$ three decades while the increase of $G^{\prime \prime}{ }_{r}$ is $\sim$ two decades in the range of interphase volume fraction studied. The increase in $G^{\prime}$, characterizing the increase in energy stored in the material when sheared, can be related to the increase in interphase volume fraction when the thickness is reduced and more precisely to the amount of anchored chains and entanglements between the bulk and the brushes. The increase in loss modulus $G^{\prime \prime}$ characterizes the presence of dissipative mechanisms that can involve large length scales, due to viscoelastic fluid deformation [6]. In this particular case, it can be associated to the sample capability for stress transfer from the interphase to the bulk. $G^{\prime}$ and $G^{\prime \prime}{ }_{r}$ follow a power law with an exponent of 0.8 and 0.5 as a function of $\phi_{\mathrm{i}}$, respectively.

It seems clear that the rheological properties are controlled by the interphase volume fraction. To conclude, we can mention the yield energy allowing the system to yield, which can be defined as $E_{y}=1 / 2 G_{o}^{\prime} \gamma_{c}^{2}$ [26], and is in this case roughly independent of the interphase volume fraction (based on the power laws previously discussed). As can be seen in Fig. S5 [37], rheological properties are independent of the number of interfaces, which tend to confirm that the interphase nature and size are identical in all systems and are governing the yield properties.

\section{B. Modeling}

The rheological behavior modeling of multilayer films is performed on the apparent complex viscosity $\eta^{*}$ (i.e., the films' response to deformation). This takes into account both $G^{\prime}$ and $G^{\prime \prime}$ contributions since different variations of moduli are reported as a function of the interphase volume fraction [Figs. 3(c) and 3(d)].

Figure 4 presents the variation of the apparent complex viscosity $\eta^{*}$ as a function of angular frequency $\omega$, in the linear domain $\left(\gamma<\gamma_{c}\right)$ for PE/PEgMA bulk (33/67 wt. \%), 25/50/ 25 wt. \% PE/PEgMA/PA6 multilayer films, and the one containing only interphase. The interphase and bulk viscosities were first adjusted with Eqs. (3) and (4) described below, and the multilayer ones using different models. These models all combine the interphase and bulk viscosities previously obtained and are summarized in Table II. A conventional viscoelastic behavior for thermoplastic is observed for PE/ PEgMA bulk (33/67 wt. \%), characterized by an extrapolated Newtonian complex viscosity $\eta_{0}^{*} \sim 1460 \mathrm{~Pa}$ s, at low angular frequencies $\omega$. At these frequencies, multilayer systems exhibit a yield behavior, in agreement with results reported by Lamnawar and Maazouz [27] in the case of a similar compatibilized bilayer film. This is due to the presence of PE-PA6 copolymer segregating at the interphase. This yield is more 

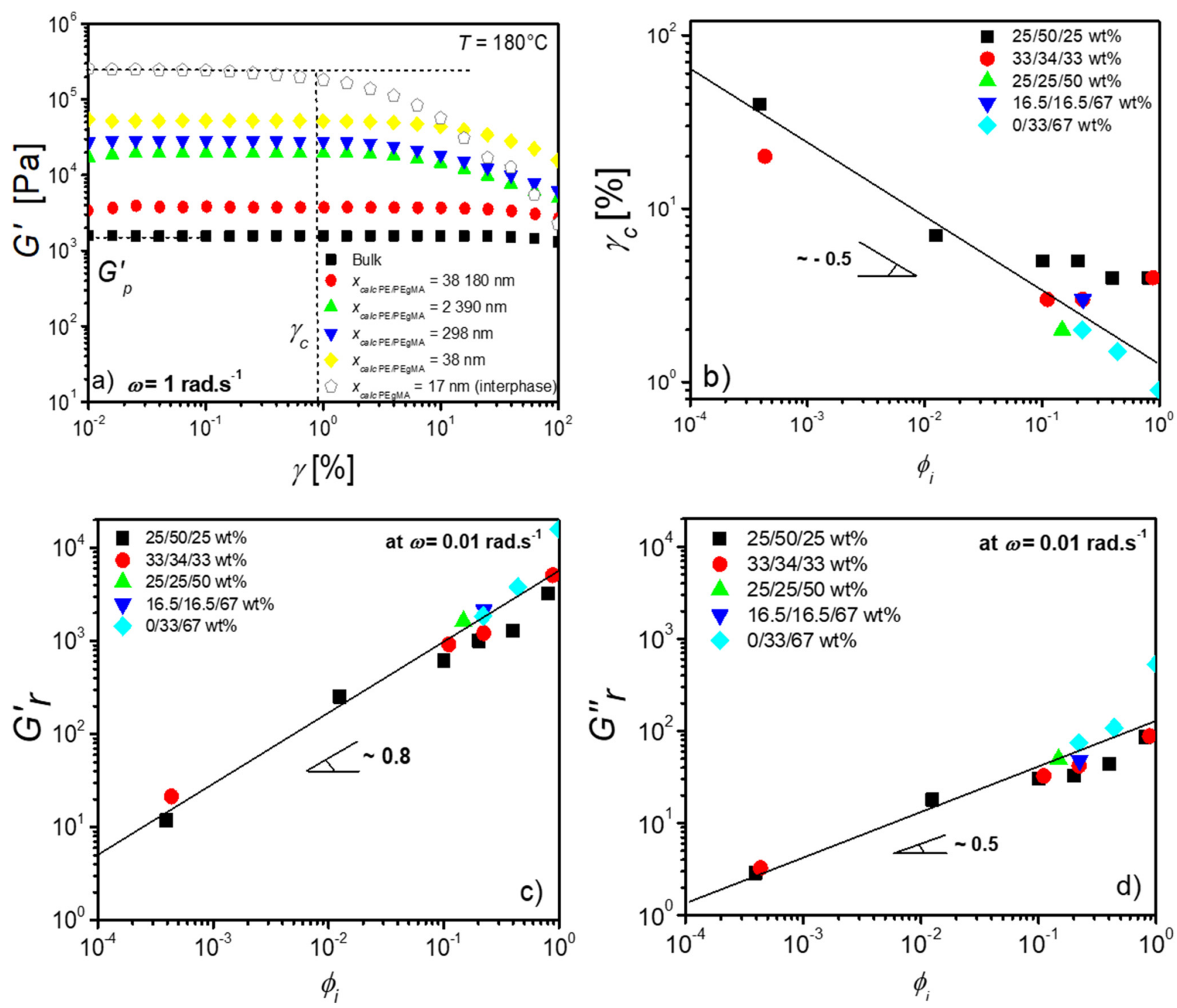

FIG. 3. Elastic modulus $G^{\prime}$ as a function of strain $\gamma$ for $33 / 67$ wt. $\%$ PE/PEgMA bulk and 25/50/25 wt. \% PE/PEgMA/PA6 multilayer films and thus containing only the interphase (a); critical strain $\gamma_{c}$ (b); relative elastic $G^{\prime}{ }_{r}$ (c); and loss $G^{\prime \prime}{ }_{r}$ (d) moduli as a function of interphase volume fraction $\phi_{\mathrm{i}}$ for all PE/PEgMA/ PA6 multilayer films.

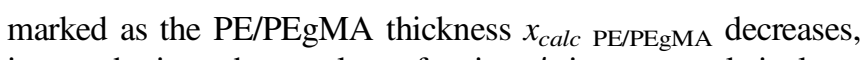
i.e., as the interphase volume fraction $\phi_{\mathrm{i}}$ increases relatively to the PE/PEgMA phase. At high angular frequencies $\omega$, all curves tend to join the PE/PEgMA bulk one. It is worth noting that for systems having layers thinner than $\sim 1 \mu \mathrm{m}$, no Newtonian plateau at low frequencies can be observed.

The next paragraph focuses on modeling the apparent complex viscosity $\eta^{*}$ of PE/PEgMA/PA6 compatibilized multilayer films.

To capture the multilayer systems' behavior, we first describe PE/PEgMA bulk and interphase viscosities $\eta_{\text {bulk }}^{*}(\omega)$ and $\eta_{\mathrm{i}}^{*}(\omega)$ as functions of angular frequency using a phenomenological Carreau-Yasuda model [25,28-30], without [for bulk (3)] and with [for interphase (4)] yield stress:

$$
\begin{gathered}
\eta_{\text {bulk }}^{*}(\omega)=\eta_{0 \text { bulk }}^{*}\left[1+\left(\lambda_{\text {bulk }} \omega\right)^{p_{\text {bulk }}}\right]^{\frac{n-1}{p_{\text {bulk }}}}, \\
\eta_{\mathrm{i}}^{*}(\omega)=\frac{\tau_{0 \mathrm{i}}}{\omega}+\eta_{0 \mathrm{i}}^{*}\left[1+\left(\lambda_{\mathrm{i}} \omega\right)^{p_{i}}\right]^{\frac{n-1}{p_{\mathrm{i}}}}
\end{gathered}
$$

with $\tau_{O}$ i, the apparent yield stress; $\eta_{0 \text { bulk }}^{*}$ and $\eta_{0 \mathrm{i}}^{*}$, the Newtonian complex viscosities; $\lambda_{\text {bulk }}$ and $\lambda_{\mathrm{i}}$, relaxation times; $p_{\text {bulk }}$ and $p_{\mathrm{i}}$, fitting parameters; and $n$, the pseudoplasticity index. $n$ has been fixed at the same value for both phases $=0.2$ because it is the value extracted from the interphase fit, and all curves appear to collapse at high angular frequencies. Moreover, it has been experimentally reported for various polymers by Graessley and can be considered a universal exponent [31]. In the model with yield stress, this classically represents the necessary stress required for the system to flow. Thus, the notion of yield stress, generally associated to the network type structure, is not evident in our case and the apparent yield observed on the viscosity curve is probably related to the slowest copolymer chain relaxation in the interphase, as suggested by Moan et al. [13]. The nonlinearities observed in Fig. 3(a) are consistent with a yield stress addition in the viscosity behavior modeling of multilayer films.

The multilayer viscosities will be modeled in the following using different combinations of bulk and interphase 


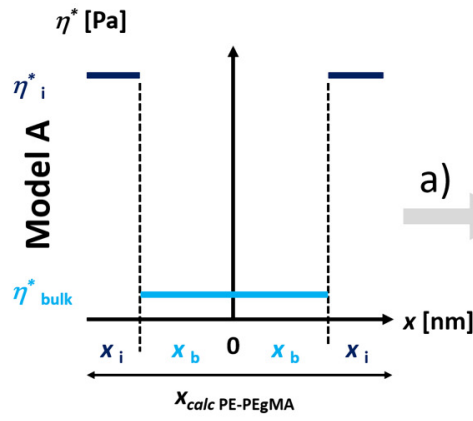

$\Delta V\left[\mathrm{~nm} \cdot \mathrm{s}^{-1}\right]$

a)
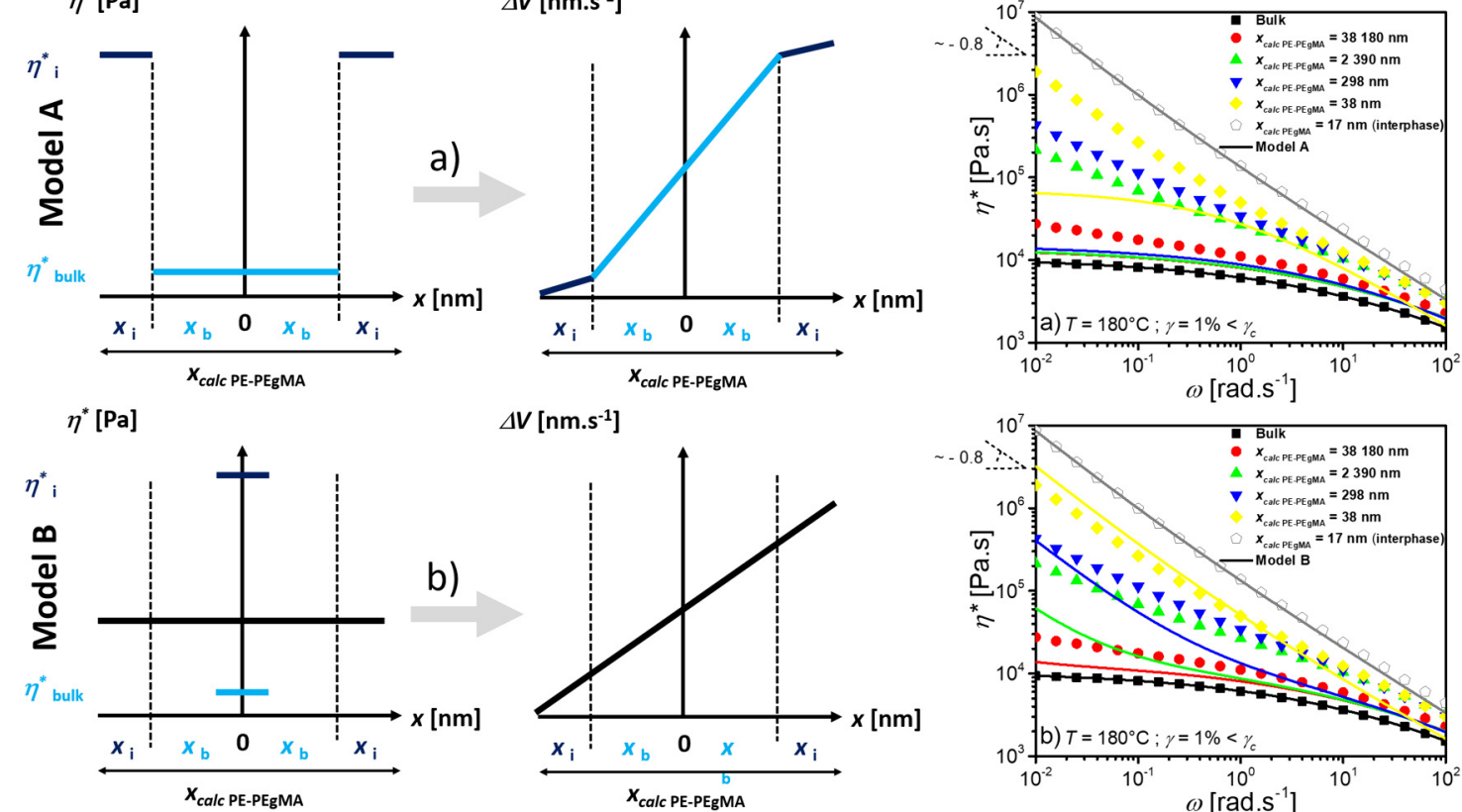

$\Delta V\left[n m . s^{-1}\right]$
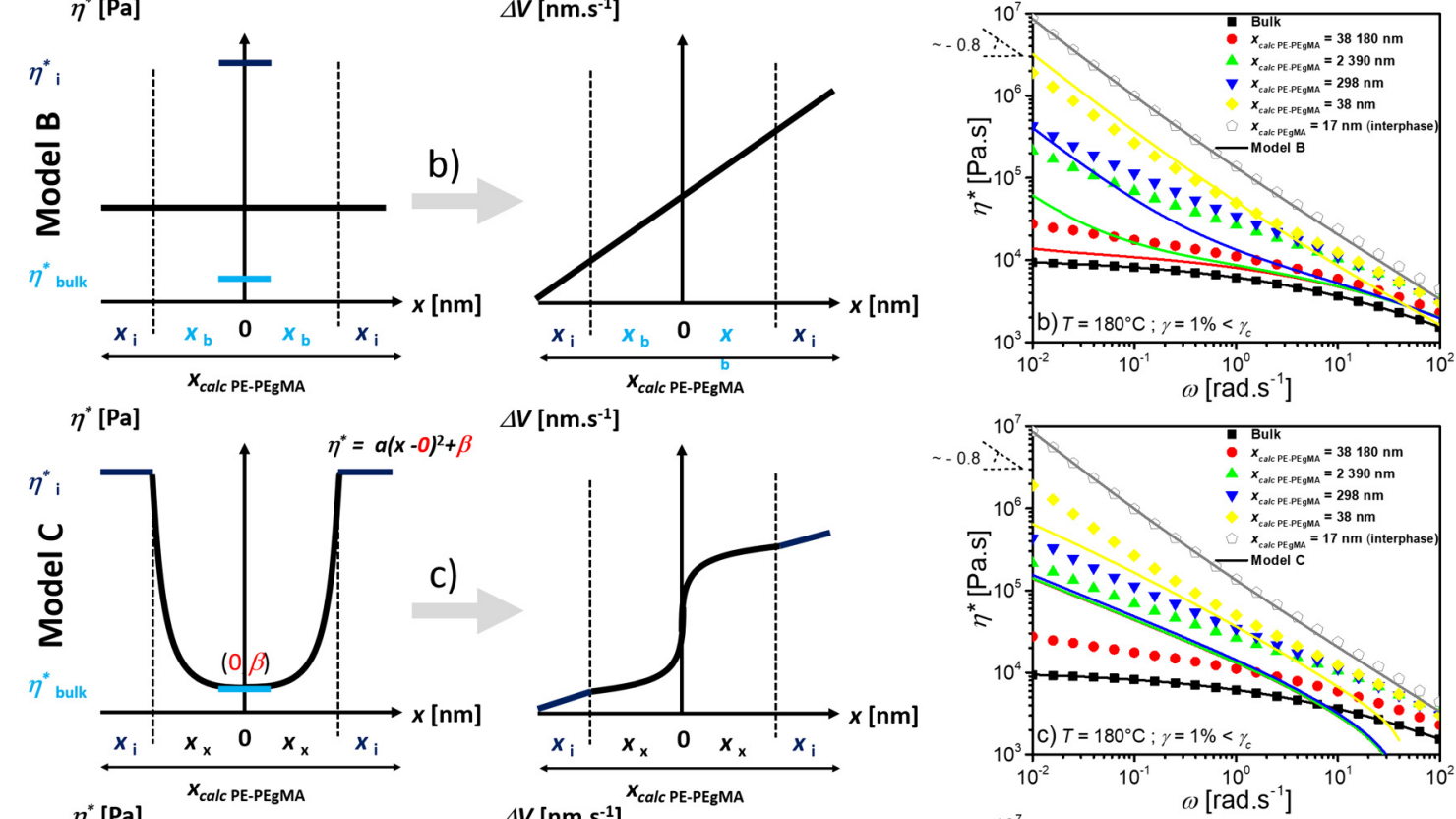

$\Delta V\left[n m . s^{-1}\right]$
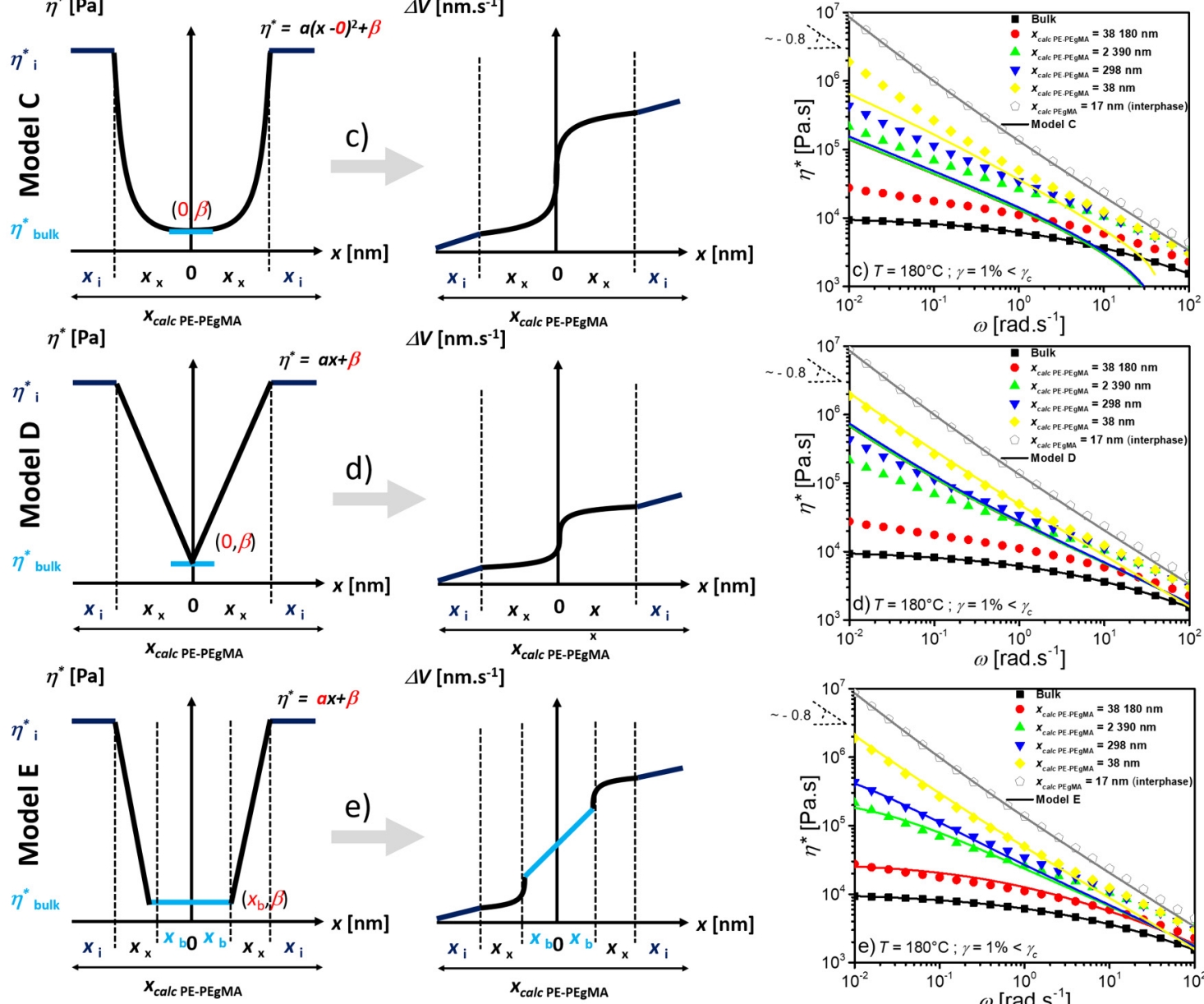

$\Delta V\left[\mathrm{~nm} \cdot \mathrm{s}^{-1}\right]$
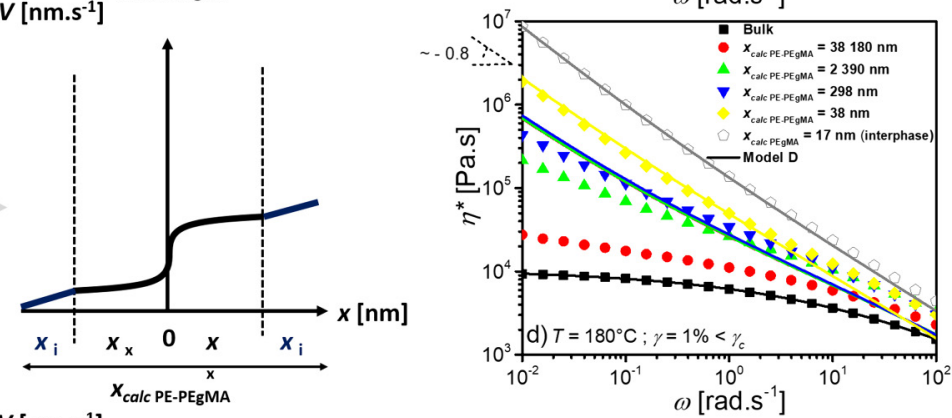

$\Delta V\left[n m \cdot s^{-1}\right]$
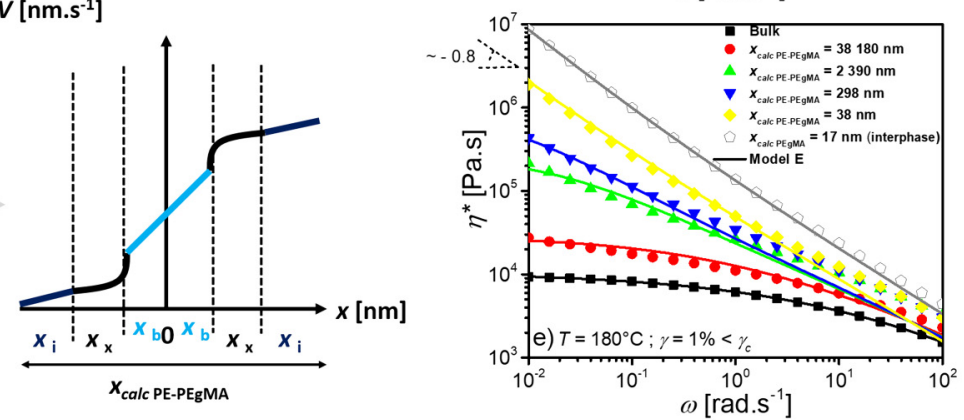

FIG. 4. Schematic of PE/PEgMA phase viscosity profile $\eta^{*}(x)$, its associated velocity gradient, and the apparent complex viscosity $\eta^{*}$ adjustment as a function of angular frequency $\omega$ for 33/67 wt. \% PE/PEgMA bulk, 25/50/25 wt. \% PE/PEgMA/PA6 multilayer films and thus containing only the interphase.

viscosities determined previously (i.e., without changing the fitting parameters obtained for them, see Table S7) [37] and compared with the experimental results.

For readability, the shear dependent viscosity $\eta^{*}(\omega)$ will be written $\eta^{*}$ in the following. In shear rheology, a serial model (addition of velocity gradient $d V$ and shear stress $\tau$ constant over all film thickness $z$ ) has to be considered to predict the apparent complex viscosity $\eta^{*}$ of multilayered films $[17,27,32,33]$. For a small thickness variation $d x$, the velocity variation $d V$ is related to the constant shear stress $\tau$ 
TABLE II. Viscosity profile $\eta^{*}(x)$ and associated apparent viscosity $\eta^{*}$ of the multilayer film.

\begin{tabular}{|c|c|c|c|}
\hline Reference & Type & $\eta^{*}(x)$ in the PE/PEgMA layer & $\eta^{*}$ of the multilayer film \\
\hline A & Constant & $\begin{array}{l}\text { For } x: 0<x<x_{\mathrm{b}} \quad \eta^{*}(x)=\eta_{\mathrm{bulk}}^{*} \\
\text { For } x: x_{\mathrm{b}}<x<x_{\mathrm{b}}+x_{\mathrm{i}}\left(=x_{\mathrm{i}}\right) \quad \eta^{*}(x)=\eta_{\mathrm{i}}^{*}\end{array}$ & $\frac{1}{\eta^{*}}=\frac{2^{N+1}}{z}\left(\frac{x_{\mathrm{b}}}{\eta_{\text {bulk }}^{*}}+\frac{x_{\mathrm{i}}}{\eta_{\mathrm{i}}^{*}}\right)$ \\
\hline B & Mixing law & For $x: 0<x<x_{\mathrm{b}}+x_{\mathrm{i}} \quad \eta^{*}(x)=\frac{x_{\mathrm{i}}}{x_{\mathrm{b}}+x_{\mathrm{i}}} \eta_{\mathrm{i}}^{*}+\frac{x_{\mathrm{b}}}{x_{\mathrm{b}}+x_{\mathrm{i}}} \eta_{\mathrm{bulk}}^{*}$ & $\frac{1}{\eta^{*}}=\frac{2^{N+1}}{z} \frac{\left(x_{\mathrm{b}}+x_{\mathrm{i}}\right)^{2}}{x_{\mathrm{b}} \eta_{\mathrm{bulk}}^{*}+x_{\mathrm{i}} \eta_{\mathrm{i}}^{*}}$ \\
\hline $\mathrm{C}$ & Parabolic & $\begin{array}{l}\text { For } x: 0<x<x_{\mathrm{x}} \quad \eta^{*}(x)=a(x-\alpha)^{2}+\beta \\
\text { For } x: x_{\mathrm{x}}<x<x_{\mathrm{x}}+x_{\mathrm{i}}\left(=x_{\mathrm{i}}\right) \quad \eta^{*}(x)=\eta_{\mathrm{i}}^{*}\end{array}$ & $\frac{1}{\eta^{*}}=\frac{2^{N+1}}{z}\left(\frac{1}{\sqrt{\beta a}} \arctan \left(\sqrt{\frac{a}{\beta}} x_{\mathrm{x}}\right)+\frac{x_{\mathrm{i}}}{\eta_{\mathrm{i}}^{*}}\right)$ \\
\hline $\mathrm{D}$ & Linear & $\begin{array}{l}\text { For } x: 0<x<x_{\mathrm{x}} \quad \eta^{*}(x)=a(x-\alpha)+\beta \\
\text { For } x: x_{\mathrm{x}}<x<x_{\mathrm{x}}+x_{\mathrm{i}}\left(=x_{\mathrm{i}}\right) \quad \eta^{*}(x)=\eta_{\mathrm{i}}^{*}\end{array}$ & $\frac{1}{\eta^{*}}=\frac{2^{N+1}}{z}\left(\frac{1}{a} \ln \left(\frac{a}{\beta} x_{\mathrm{x}}+1\right)+\frac{x_{\mathrm{i}}}{\eta_{\mathrm{i}}^{*}}\right)$ \\
\hline $\mathrm{E}$ & Constant + Linear & $\begin{array}{c}\text { For } x: 0<x<x_{\mathrm{b}} \quad \eta^{*}(x)=\eta_{\text {bulk }}^{*} \\
\text { For } x: x_{\mathrm{b}}<x<x_{\mathrm{b}}+x_{\mathrm{x}}\left(=x_{\mathrm{xfree}}\right) \quad \eta^{*}(x)=a x+b \\
\text { For } x: x_{\mathrm{b}}+x_{\mathrm{x}}<x<x_{\mathrm{b}}+x_{\mathrm{x}}+x_{\mathrm{i}}\left(=x_{\mathrm{i}}\right) \quad \eta^{*}(x)=\eta_{\mathrm{i}}^{*}\end{array}$ & $\frac{1}{\eta^{*}}=\frac{2^{N+1}}{z}\left(\frac{x_{\mathrm{b}}}{\eta_{\mathrm{bulk}}^{*}}+\frac{1}{a} \ln \left(\frac{a\left(x_{\mathrm{b}}+x_{\mathrm{x}}\right)+\beta}{a x_{\mathrm{b}}+\beta}\right)+\frac{x_{\mathrm{i}}}{\eta_{\mathrm{i}}^{*}}\right)$ \\
\hline
\end{tabular}

and the viscosity at distance $x, \eta^{*}(x)$,

$$
\mathrm{d} V=\frac{\tau}{\eta^{*}(x)} \mathrm{d} x
$$

and the total variation of velocity $\Delta V$ is

$$
\Delta V=\int_{0}^{z} \mathrm{~d} V=\int_{0}^{z} \frac{\tau}{\eta^{*}(x)} \mathrm{d} x
$$

The total shear rate $\dot{\gamma}$ corresponds to the total velocity gradient $\Delta V$ on the total film thickness $z$. It can be expressed as the ratio between the shear stress $\tau$ (constant in the sample) and the apparent complex viscosity $\eta^{*}$ of the multilayered film,

$$
\dot{\gamma}=\frac{\tau}{\eta^{*}}=\frac{\Delta V}{z} .
$$

Substituting (6) in (7) leads to the following equations:

$$
\begin{gathered}
\frac{\tau}{\eta^{*}}=\frac{\int_{0}^{z} \frac{\tau}{\eta^{*}(x)} \mathrm{d} x}{z}, \\
\frac{\tau}{\eta^{*}}=\frac{\tau}{z} \int_{0}^{z} \frac{1}{\eta^{*}(x)} \mathrm{d} x, \\
\frac{1}{\eta^{*}}=\frac{1}{z} \int_{0}^{z} \frac{1}{\eta^{*}(x)} \mathrm{d} x .
\end{gathered}
$$

The total film thickness $z$ can be divided into the sum of the individual $x_{\mathrm{b}}, x_{\mathrm{x}}, x_{\mathrm{i}}\left(2^{N+1}\right.$ layers $)$, and $x_{\mathrm{PA} 6}\left(2^{N}\right.$ layers $)$ layer thicknesses as follows:

$$
z=2^{N+1}\left(x_{\mathrm{b}}+x_{\mathrm{x}}+x_{\mathrm{i}}\right)+2^{N} x_{\mathrm{PA} 6},
$$

where $N$ is the number of LME as previously defined. The integral on total thickness $0<x<z$ (10) can be split into two parts according to the Chasles relationship over $x$ as follows:

$$
\frac{1}{\eta^{*}}=\frac{1}{z}\left(2^{N+1} \int_{0}^{x_{\mathrm{i}}+x_{\mathrm{x}}+x_{\mathrm{b}}} \frac{1}{\eta^{*}(x)} \mathrm{d} x+2^{N} \int_{x_{\mathrm{i}}+x_{\mathrm{x}}+x_{\mathrm{b}}}^{z} \frac{1}{\eta^{*}(x)} \mathrm{d} x\right)
$$

PA6 is in the solid state $\left(x_{\mathrm{i}}+x_{\mathrm{x}}+x_{\mathrm{b}}<x<x_{\mathrm{PA} 6}\right)$, and it does not contribute on apparent film viscosity $\eta^{*}$ in shear rheology. The second part of the integral (12) can then be neglected, which leads to the following equation:

$$
\frac{1}{\eta^{*}}=\frac{2^{N+1}}{z} \int_{0}^{x_{\mathrm{i}}+x_{\mathrm{x}}+x_{\mathrm{b}}} \frac{1}{\eta^{*}(x)} \mathrm{d} x
$$

Different viscosity profiles for PE/PEgMA phases have been proposed to go continuously through high interphase $\eta_{\mathrm{i}}^{*}$ to low bulk viscosity $\eta_{\text {bulk }}^{*}$ within the layer thickness. Table II and Fig. 4 summarize the different model (A-E) equations and data adjustments, corresponding to calculations starting from Eq. (13) and detailed in Supporting Information for the different viscosity profiles $\eta^{*}(x)$ selected: (a) a serial model with constant bulk $\eta_{\text {bulk }}^{*}$ and interphase $\eta_{\mathrm{i}}^{*}$ viscosity without interaction between melt polymer and interphase $[17,32]$, (b) simple weighted average mixing law between bulk $\eta_{\text {bulk }}^{*}$ and interphase $\eta_{\mathrm{i}}^{*}$ viscosity [18], (c) parabolic and (d) linear variation of viscosity from $\eta_{\mathrm{i}}^{*}$ to $\eta_{\text {bulk }}^{*}$ in the middle of the layer $(x=0)$, and (e) a combination of bulk $\eta_{\text {bulk }}^{*}$ and interphase $\eta_{\mathrm{i}}^{*}$ constant viscosity and a linear viscosity variation on the free $x_{\mathrm{x}}$ thickness. For all models, the viscosity profile over the nanometric interphase thickness $\left(x_{\mathrm{i}}=15 \mathrm{~nm}\right)$ is assumed constant. The schematic variation of the viscosity profiles (a-e) over the PE/PEgMA layer, their associated velocity gradient $\Delta V$, and their respective viscosity data adjustments are presented in Fig. 4.

The most reasonable physical model of the viscosity profile $\eta^{*}(x)$ through the PE/PEgMA ( $x_{\text {calc }}$ PE/PEgMA $)$ layer with the compatibilized interphase will be discussed, in the light of the best data fitting. The conventional serial model [Fig. 4(a)], without interaction between the interphase and the bulk of PE/PEgMA largely, underestimates the apparent complex viscosity values $\eta^{*}$ for all multilayer films. Moreover, it does not capture the rheological behavior as a function of angular frequency $\omega$, indicating that the PE/ PEgMA layer viscosity profile is more complex.

As a first approximation, the simple average mixing law between the interphase and bulk viscosities [Fig. 4(b)] roughly captures the curve shapes but not perfectly their viscosity level. A parabolic [Fig. 4(c)] abrupt transition from interphase $\eta_{\mathrm{i}}^{*}$ to bulk $\eta_{\text {bulk }}^{*}$ viscosities until the middle of the PE/PEgMA layer $(x=0)$ is not satisfactory. The linear one correctly fits the data with the thinnest PE/PEgMA layers (up 
to few hundreds nanometers for $x_{\text {calc }}$ PE/PEgMA ) but largely overestimates those of the other films [Fig. 4(d)], which suggests the interphase influences the bulk phase only up to a certain critical distance. Hence, we proposed an original viscosity profile, with a linear transition over a free $x_{\mathrm{x}}$ thickness between two constant interphase $\left(\eta_{\mathrm{i}}^{*}\right)$ and bulk $\left(\eta_{\text {bulk }}^{*}\right)$ viscosities on $x_{\mathrm{i}}=15 \mathrm{~nm}$ and $x_{\mathrm{b}}$ thicknesses, respectively. This model using an additional fitting parameter $\left(x_{\mathrm{x}}\right)$ enables the prediction of the rheological data of all multimicro- and multinanolayered films over quasi-all angular frequency $\omega$ range [Fig. 4(e)]. At high frequencies, the model (and all others) slightly underestimates the rheological signal.

Figure 5 shows, for all PE/PEgMA/PA6 film concentrations, the ratio of the free parameter $x_{\mathrm{x}}$ (characterizing the thickness of the linear viscosity transition in model E) over half the PE/PEgMA layer thickness excluding the interphase, i.e., $x_{\mathrm{x}} /\left(x_{\mathrm{b}}+x_{\mathrm{x}}\right)$, as a function of $x_{\mathrm{b}}+x_{\mathrm{x}}$. Figure 5 shows that this ratio is close to 1 up to a thickness of $\sim 1 \mu \mathrm{m}$ and then progressively decreases, meaning that $x_{\mathrm{b}}$ becomes nonnegligible only for the largest layers and above $\sim 1 \mu \mathrm{m}$ (the values for $x_{\mathrm{b}}$ and $x_{\mathrm{x}}$ can be seen in Fig. S8) [37]. In other words, the linear profile, as shown in Fig. 4(d), can also describe the rheological data for layer thicknesses below $1 \mu \mathrm{m}$. However, at this point, the interphase influence starts to vanish, and for thicker layers, an unperturbed bulklike phase has to be taken into account to capture the rheological behavior: it is clear that for these systems model $\mathrm{D}$ does not capture the experimental results [see Fig. 4(d) where blue, green, and red curves coincide]. The value of $1 \mu \mathrm{m}$ can then be associated to the typical distance over which the interphase strongly influences the melt due to viscoelastic fluid deformation. This relatively large value suggests that the mechanisms responsible for the interphase impact on the rheological properties are not molecular in nature but could rather be due, as mentioned earlier, to long scale dissipative mechanisms enabling stress transfer between anchored brushes and the interpenetrated melt polymer. Such long scale dissipative mechanisms have, for example, been

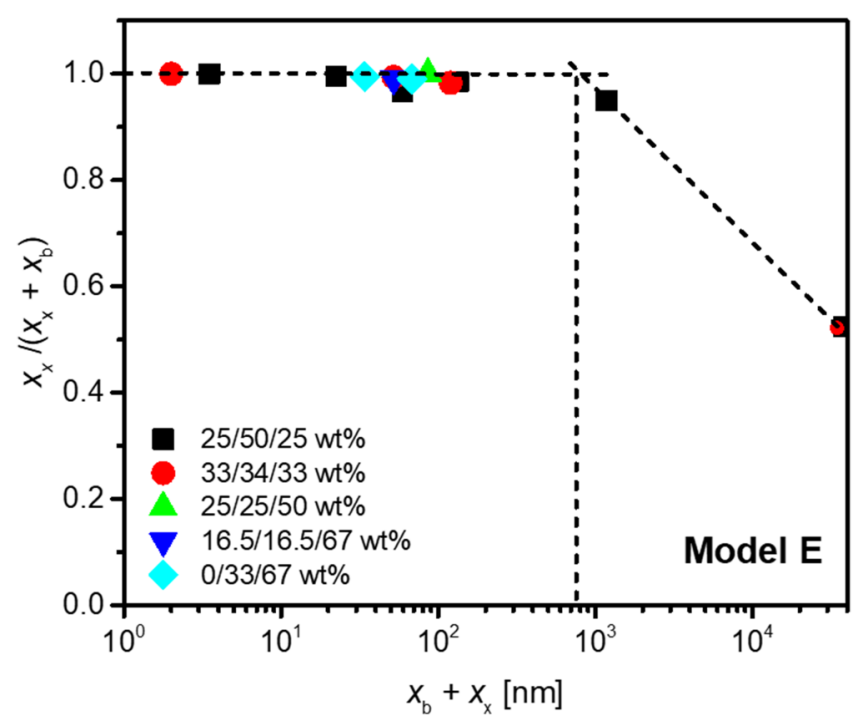

FIG. 5. Variation of $x_{\mathrm{x}} /\left(x_{\mathrm{b}}+x_{\mathrm{x}}\right)$ as a function of $x_{\mathrm{b}}+x_{\mathrm{x}}$ thickness for multilayer films with different PE/PEgMA/PA6 concentrations in model E. observed recently in the case of crack propagation in double network elastomers, via the use of mechanoluminescent molecules [34,35].

Even if further studies are needed to conclude on a particular mechanism, two potential candidates for explaining the observed behavior are as follows: (i) the interaction between the bulk molecules and the anchored brushes, expected to diffuse into the bulk perturbing the flow with its associated increase of the apparent viscosity, and (ii) the bulk in which flow could be perturbed by the intrinsic roughness of the anchored brushes interface [36]. It would be expected for the former mechanism related to molecular interaction diffusion to have a characteristic length smaller than the one experimentally identified (typically on the order of a few molecular lengths at most, i.e., $\lesssim 100 \mathrm{~nm}$ ). The second one, however, due to kinematic perturbation diffusion in the flowing bulk induced by interface roughness, may have a very large characteristic penetration length. Our future works will aim at analyzing these mechanisms in further details.

\section{CONCLUSION}

The rheological behavior of coextruded multimicro- and multinanolayered films is clearly controlled by the layer thickness and as a consequence by the interphase volume fraction. The compatibilized interphase contributes to both elastic and loss moduli by direct chemical bonding and dissipative mechanisms, respectively. The crucial role of polymer compatibilization at the nanoscale in such systems has been highlighted using simple macroscopic rheological tests. We have proposed a consistent physical modeling of the viscosity profile in each layer for all the systems studied, covering five concentrations, a number of interfaces varying from 2 to 1024 , and layer thicknesses from over $30 \mu \mathrm{m}$ to less than $20 \mathrm{~nm}$, using only one fitting parameter. In this model, the fitting parameter is the thickness over which viscosity decreases linearly from the interphase to the bulk one. This parameter increases with the layer thickness up to layer thicknesses of $\sim 1 \mu \mathrm{m}$ before a bulk layer has to be added in the viscosity profile. This critical thickness, having a value quite surprisingly high, can be seen as the length over which the interphase influences the rheological behavior.

\section{ACKNOWLEDGMENTS}

The authors acknowledge the French National Research Agency (ANR, project IMMUNE No. 16-CE08-0035, coordinator Stéphane Marais) for its financial support. Liliane Léger and Dr. Frederic Restagno are acknowledged for their fruitful discussions on this work. The authors are grateful to Emmanuel Richaud and to Dr. Nicolas Longerias from PeakExpert (Tours, France) for gas permeation chromatography (GPC) analysis, to Dr. Clément Freymond for the FTIR measurements, and to Dr. Ilias Iliopoulos for helpful comments on FTIR and NMR. The authors would like to thank Anne Grandmontagne for her help in coextrusion. They also thank Dr. Michel Cloître and Dr. Sophie Norvez from the Ecole Supérieure de Physique et de Chimie Industrielle (ESPCI, Paris, France) for providing access to the ultracryomicrotome facility. 


\section{REFERENCES}

[1] Utracki, L. A., Commercial Polymer Blends (Springer, Boston, 1998).

[2] Sundararaj, U., and C. W. Macosko, "Drop breakup and coalescence in polymer blends: The effects of concentration and compatibilization," Macromolecules 28, 2647-2657 (1995).

[3] Yukioka, S., and T. Inoue, "Ellipsometric analysis on the in situ reactive compatibilization of immiscible polymer blends," Polymer 35, 1182-1186 (1994).

[4] Tang, T., and B. Huang, "Interfacial behaviour of compatibilizers in polymer blends," Polymer 35, 281-285 (1994).

[5] Creton, C., E. J. Kramer, H. R. Brown, and C. Y. Hui, "Adhesion and fracture of interfaces between immiscible polymers: From the molecular to the continuum scale," Adv. Polym. Sci. 156, 53-136 (2002).

[6] Léger, L., and C. Creton, "Adhesion mechanisms at soft polymer interfaces," Philos. Trans. R. Soc. A Math. Phys. Eng. Sci. 366, 1425-1442 (2008).

[7] Brown, H. R., "The adhesion of polymers: Relations between properties of polymer chains and interface toughness," J. Adhes. 82, 1013-1032 (2006).

[8] Ponting, M., A. Hiltner, and E. Baer, "Polymer nanostructures by forced assembly: Process, structure, and properties," Macromol. Symp. 294, 19-32 (2010).

[9] Bironeau, A., T. Salez, G. Miquelard-Garnier, and C. Sollogoub, "Existence of a critical layer thickness in PS/PMMA nanolayered films," Macromolecules 50, 4064-4073 (2017).

[10] Du, Q., G. Jiang, J. Li, and S. Guo, "Adhesion and delamination failure mechanisms in alternating layered polyamide and polyethylene with compatibilizer," Polym. Eng. Sci. 50, 1111-1121 (2010).

[11] Graebling, D., R. Muller, and J. F. Palierne, "Blends in the melt. Interpretation of data with a model of," Macromolecules 26, 320-329 (1993).

[12] Riemann, R.-E., H.-J. Cantow, and C. Friedrich, "Interpretation of a new interface-governed relaxation process in compatibilized polymer blends," Macromolecules 30, 5476-5484 (1997).

[13] Moan, M., J. Huitric, P. Médéric, and J. Jarrin, "Rheological properties and reactive compatibilization of immiscible polymer blends," J. Rheol. 44, 1227-1245 (2000).

[14] Levitt, L., C. W. Macosko, T. Schweizer, and J. Meissner, "Extensional rheometry of polymer multilayers: A sensitive probe of interfaces," J. Rheol. 41, 671-685 (1997).

[15] Lu, B., K. Lamnawar, and A. Maazouz, "Influence of in situ reactive interphase with graft copolymer on shear and extensional rheology in a model bilayered polymer system," Polym. Test. 61, 289-299 (2017).

[16] Jordan, A. M., B. Lee, K. Kim, E. Ludtke, O. Lhost, S. A. Jaffer, F. S. Bates, and C. W. Macosko, "Rheology of polymer multilayers: Slip in shear, hardening in extension," J. Rheol. 63, 751-761 (2019).

[17] Zhao, R., and C. W. Macosko, "Slip at polymer-polymer interfaces: Rheological measurements on coextruded multilayers," J. Rheol. 46, 145-167 (2002).

[18] Beuguel, Q., A. Guinault, L. Léger, F. Restagno, C. Sollogoub, and G. Miquelard-garnier, "Nanorheology with a conventional rheometer: Probing the interfacial properties in compatibilized multinanolayer polymer films," ACS Macro Lett. 8, 1309-1315 (2019).

[19] Samay, G., T. Nagy, and J. L. White, "Grafting maleic anhydride and comonomers onto polyethylene,” J. Appl. Polym. Sci. 56, 1423-1433 (1995).

[20] Garmabi, H., N. R. Demarquette, and M. R. Kamal, "Effect of temperature and compatibilizer on interfacial tension of PE/PA-6 and PP/ EVOH,” Int. Polym. Process. 13, 183-191 (1998).
[21] Bironeau, A., Films multinanocouches de polymères amorphes coextrudés: élaboration, caractérisation et stabilité des nanocouches, Ph.D. thesis, Ecole Nationale Supérieure des Arts et Métiers, Paris, 2016, 1-247.

[22] Kiparissoff-Bondil, H., S. Devisme, D. Rauline, F. Chopinez, F. Restagno, and L. Léger, "Evidences for flow-assisted interfacial reaction in coextruded PA6/PP/PA6 films," Polym. Eng. Sci. 59, E44-E50 (2019).

[23] Bironeau, A., J. Dirrenberger, C. Sollogoub, G. Miquelard-Garnier, and S. Roland, "Evaluation of morphological representative sample sizes for nanolayered polymer blends," J. Microsc. 264, 48-58 (2016).

[24] Vuong, S., N. Chedozeau, J. Guilment, L. Léger, and F. Restagno, "Quantitative determination of interfacial copolymers from co-extruded films," Colloid. Surf. A 529, 261-267 (2017).

[25] Macosko, C. W., Rheology: Principles, Measurements and Applications (Wiley-VCH, New York, 1994).

[26] Aubry, T., T. Razafinimaro, and P. Médéric, "Rheological investigation of the melt state elastic and yield properties of a polyamide-12 layered silicate nanocomposite,” J. Rheol. 49, 425-440 (2005).

[27] Lamnawar, K., and A. Maazouz, "Rheology and morphology of multilayer reactive polymers: Effect of interfacial area in interdiffusion/reaction phenomena," Rheol. Acta 47, 383-397 (2008).

[28] Bird, B. R., and P. J. Carreau, "A nonlinear viscoelastic model for polymer solutions and melts-I," Chem. Eng. Sci. 23, 427-434 (1968).

[29] Carreau, P. J., I. F. MacDonald, and R. B. Bird, "A nonlinear viscoelastic model for polymer solutions and melts-II," Chem. Eng. Sci. 23, 901-911 (1968).

[30] Yasuda, K., R. C. Armstrong, and R. E. Cohen, "Shear flow properties of concentrated solutions of linear and star branched polystyrenes," Rheol. Acta 20, 163-178 (1981).

[31] Graessley, W. W., The Entanglement Concept in Polymer Rheology (Springer-Verlag, Berlin, 1974).

[32] Lin, C.-C., "A mathematical model for viscosity in capillary extrusion of two-component polyblends," Polym. J. 11, 185-192 (1979).

[33] Carriere, C. J., and R. Ramanathan, "Multilayer rheology: A comparison of experimental data with modeling of multilayer shear flow," Polym. Eng. Sci. 35, 1979-1984 (1995).

[34] Ducrot, E., Y. Chen, M. Bulters, R. P. Sijbesma, and C. Creton, "Toughening elastomers with sacrificial bonds and watching them break," Science 344, 186-189 (2014).

[35] Millereau, P., E. Ducrot, J. M. Clough, M. E. Wiseman, H. R. Brown, R. P. Sijbesma, and C. Creton, "Mechanics of elastomeric molecular composites," Proc. Natl. Acad. Sci. U.S.A. 115, 9110-9115 (2018).

[36] Ammar, A., C. Ghnatios, F. Delplace, A. Barasinski, J. Duval, E. Cueto, and F. Chinesta, "On the effective conductivity and the apparent viscosity of a thin rough polymer interface using PGD-based separated representations," Int. J. Numer. Methods Eng. (published online 2020).

[37] See supplementary material at https://doi.org/10.1122/1.5143899 for the rheological properties of the polymers used in the study at the extrusion temperature (Fig. S1); a table detailing the layer thicknesses for all the films studied (Table S2); the influence of sample preparation on the rheological response (Fig. S3); the elastic and loss moduli of the multilayer films as a function of angular frequency (Fig. S4); the critical strain and relative (elastic and loss) moduli as a function of layer thickness (Fig. S5); the conservation of the layered structure after the rheological test (Fig. S6); details of the calculation for each model; and thickness values extracted from model E (Fig. S7). 